\title{
Universal Fluctuations of the Random Lasing Threshold in a Sample of a Finite Area
}

\author{
V. M. Apalkov and M. E. Raikh
}

Department of Physics, University of Utah, Salt Lake City, Utah 84112

\begin{abstract}
We consider the random lasing from a weakly scattering medium and demonstrate that the distribution of the threshold gain over the ensemble of statistically independent finite-size samples is universal. Universality stems from the facts that: (i) lasing threshold in a given sample is determined by the highestquality mode of all the random resonators present in the sample, and (ii) the areal density of the random resonators decays sharply with the quality factor of the mode that they trap. We find analytically the shape of the universal distribution function of the lasing threshold. The shape of this function is governed by a single dimensionless parameter, $\beta$. This parameter increases as a power law with $\ln S$, where $S$ is the sample area (length, volume), and decreases as a power law with disorder strength. The powers depend on the microscopic mechanism of the light trapping. As a result, the distribution of the thresholds narrows with $S$ and broadens with the disorder strength.
\end{abstract}

\section{Introduction}

During the last five years the phenomenon of coherent random lasing has been observed in a wide variety of disordered media. These include semiconductor powders ${ }^{1}$, polymer films ${ }^{2}$, dye-infiltrated opals ${ }^{2}$, etc. The fact, that beyond a certain optical excitation threshold, the random media emits a coherent light (this fact was demonstrated in the photon statistics experiments ${ }^{3,4}$ ) suggests that certain disorder configurations in the medium (random resonators ${ }^{5}$ ) are capable of "trapping" the light, thus assuming the role of the conventional Fabry-Perot resonators. These configurations are sparse, since on average the light propagation in the media is diffusive, i.e. the condition $k l \gg 1$ is met, where $k$ is the wave number and $l$ is the mean free path. As it was demonstrated analytically, ${ }^{5}$ even for a given $k l$-value, the ability of the medium to form resonators depends strongly on the character of the disorder. For example, from the point of view of trapping the light, smooth disorder is much more favorable than the point-like scatterers ${ }^{5}$ (see also Ref. 6). It is also clear on the general grounds, that two statistically identical disordered samples of a finite size have different thresholds for coherent lasing. This is because the most "capable" random resonators, that are present in each sample and determine its lasing threshold, have different quality factors ${ }^{7}$. Therefore, the study of the threshold dependence on the sample size requires a statistical description.

Consider for concreteness a polymer film. Then the role of the sample size is 
simply played by the area, $S$, of the excitation spot. Quantitatively, the change of the threshold excitation intensity, $I$, with $S$ should be characterized by the evolution of the distribution function, $F_{S}(I)$, of the thresholds over many non-overlapping spots. Namely, the position of maximum of $F_{S}(I)$, that describes the threshold of a typical sample, should shift towards lower $I$ with increasing $S$, whereas the width, that carries the information about the spread of the thresholds in different samples, should decrease with $S$.

The main message of the present paper is that the shape of the function $F_{S}(I)$ is universal and is given by

$$
F_{S}(I)=\frac{\beta_{s}}{I}\left(\frac{I}{I_{S}}\right)^{-\beta_{s}} \exp \left[-\left(\frac{I}{I_{S}}\right)^{-\beta_{s}}\right],
$$

where the typical value, $I_{S}$, is related to the sample area $S$ as follows

$$
I_{S} \propto \exp \left\{-\left[\frac{\ln \left(S / S_{0}\right)}{G}\right]^{1 / \lambda}\right\}
$$

where the parameters $\lambda>1$ and $G$ are determined by the intrinsic properties of the disordered medium and are independent of $S$; the area $S_{0}$ is a typical area of a random resonator. These two parameters play a different role: while $\lambda$ is determined exclusively by the shape of the disorder correlator, $G$ is a measure of the disorder strength. The dependence of $\beta_{s}$ on $S$ is logarithmical, namely, $\beta_{s} \propto\left[\ln \left(S / S_{0}\right)\right]^{(\lambda-1) / \lambda}$. Most importantly, parameter $\beta_{s}$ decreases with the disorder parameter $(k l)^{-1}$ as a power law, i.e. for a weakly scattering medium we have $\beta_{s} \gg 1$. The concrete value of the exponent $\partial \ln \beta / \partial \ln (k l)$ depends on the microscopic properties of the disorder.

Experimentally, establishing the form of the distribution function amounts to dividing of the measured values of $I$ into "bins" and constructing a hystogram from these bins. In this regard, it is convenient to fit the data to the theory using the momenta of the distribution, calculated with the help of experimentally determined hystogram. In particular, as it easily follows from Eq. (1), the first moment is given by

$$
\langle I\rangle=I_{S} \Gamma\left(1-\beta_{s}^{-1}\right),
$$

where $\Gamma(x)$ is the $\Gamma$-function. It can be also shown from Eq. (1) that the average logarithm of $I /\langle I\rangle$ is given by

$$
\langle\ln I\rangle-\ln \langle I\rangle=\phi\left(\beta_{s}\right),
$$


where the function $\phi(u)$ is defined as

$$
\phi(u)=\frac{\gamma}{u}-\ln \left[\Gamma\left(1-u^{-1}\right)\right] .
$$

Here $\gamma \approx 0.5772$ is the Euler's constant. The dependence $\phi(u)$ is shown in Fig. 1 . It diverges as $\ln (u-1)$ in the limit $u \rightarrow 1$ and approaches zero as $\phi(u) \approx-\frac{\pi^{2}}{12 u^{2}}$ for $u \gg 1$. The relations Eq. (3) and Eq. (5) are sufficient to restore the parameters $I_{S}$ and $\beta_{s}$ from experimentally measured hystogram. Indeed, calculating from the data the values of $\langle I\rangle$ and $\langle\ln I\rangle$, allows first to determine the parameter $\beta_{s}$ from Fig. 1, and subsequently, the parameter $I_{S}$ from Eq. (3). Comparison of the experimental distribution with theoretical prediction Eq. (1) allows to test the basic underlying assumption about the statistical independence of different random resonators. On the quantitative level, it allows to determine $\lambda$, which is strongly sensitive to the microscopic arrangement of the disorder-induced resonator.

The distribution function Eq. (1) is plotted in Fig. 2 for three values of $\beta_{s}$. Firstly, we observe that for smallest $\beta_{s}$ the distribution $F_{S}(I)$ is broad and strongly asymmetric. It has a long tail towards the high thresholds and falls off abruptly towards low thresholds. Our main qualitative prediction is that in experiments on random lasers the distribution of thresholds over samples must have a distinctive shape similar to that of the curve 1 in Fig. 2. Indeed, experimentally, the lasing is observed only when the disorder is strong enough. The realistic values ${ }^{5}$ of the disorder parameter $k l$ are $\sim 5$. On the other hand, strong disorder translates into the values of the parameter $\beta_{s}$ that are $\sim 1$.

As disorder decreases, leading to the rise of the average threshold, the distribution $F_{S}(I)$ becomes progressively narrow and symmetric. For highest $\beta_{s}=6$ (curve 3 in Fig. 2) this distribution is close to gaussian, $F_{S}(I) \propto \exp \left[-\frac{\beta_{s}^{2}}{2}\left(\frac{I-I_{S}}{I_{S}}\right)^{2}\right]$. We emphasize, that the distribution Eq. (1) is derived under the assumption that all samples (excitation spots) are statistically independent. Therefore, experimental observation of a strongly asymmetric distribution of thresholds over samples would be a confirmation that the underlying disordered medium is homogeneous on average, i.e. it does not contain large-size fluctuations of technological origin (larger than the sample size). The remaining part of the paper is organized as follows. In Sect. II we demonstrate that, with accuracy of a numerical factor, the basic characteristics of the distribution Eq. (1) can be found from a simple reasoning. In Sect. III we provide a rigorous derivation of Eq. (1). In Sect. IV we justify the validity of the basic assumption behind Eq. (1) concerning the functional form of the density of random resonators. In Sect. V. we discuss the tests of the applicability of the 
distribution (1) based on the relations between the different cumulants. The details of the calculation of these cumulants are presented in the Appendix.

\section{Qualitative consideration}

The position of maximum and the width of the distribution function $F_{S}$ can be found following the qualitative consideration of Refs. 8-10. Denote with $\rho(Q)$ the areal density of disorder-induced resonators with the quality factor $Q$. This density represents the statistical weight of disorder configurations that are capable of trapping a mode with an anomalously small relative linewidth, $Q^{-1} \ll 1$. According to the definition, $\rho(Q)$ is the characteristics of an infinite medium. It describes the ability of the medium to trap the light wave for anomalously long time (namely, for $Q$ wave periods). Note, that $\rho(Q)$ is a meaningful characteristics of the medium in two and three dimensions, where the light propagation is diffusive on average.

The function $\rho(Q)$ was calculated analytically for certain models of disorder in Refs. 5, 11, 12. The related quantity was also invoked in Ref. 13 for the analysis of simulation results. Similar function was studied in relation to current relaxation in disordered conductors ${ }^{14-17}$. All analytical results obtained within different approaches and for different models of disorder yield the same general form of the density $\rho(Q)$ in the large- $Q$ limit, namely

$$
\left.\rho(Q)\right|_{Q \gg 1}=S_{0}^{-1} \exp \left[-G(\ln Q)^{\lambda}\right]
$$

where, within a numerical factor, $G$ is related to the average "conductance", $k l$, of the disordered medium as $G=(k l)^{\mu}$, with $\mu$ being a model-dependent exponent. The power, $\lambda$, of the $\ln Q$ in Eq. (6) does not depend on the $k l$-value. It is determined exclusively by the form of the correlation function of the disorder.

The position of the maximum of the threshold distribution follows from a simple observation that the number of highest- $Q$ resonators, responsible for lasing in a typical sample, is $\sim 1$. This observation can be expressed analytically as

$$
S \rho\left(Q_{S}\right) \approx 1
$$

Eq. (7) can be viewed as an equation for $Q_{S}$, which is the quality factor of the highest- $Q$ resonator present in a typical sample. Substituting the form (6) of $\rho(Q)$ into Eq. (7), we obtain

$$
Q_{S}=\exp \left\{\left[\frac{\ln \left(S / S_{0}\right)}{G}\right]^{1 / \lambda}\right\}
$$


In order to estimate the width of the distribution, we introduce the auxiliary quantity $\tilde{Q}_{S}$, defined as the maximal $Q$-factor of a resonator which is present in almost all the samples. Since the density $\rho(Q)$ grows rapidly with $Q$, the definition of $\tilde{Q}_{S}$ can be quantified by the condition

$$
S \rho\left(\tilde{Q}_{S}\right) \approx 2,
$$

meaning that if the number of resonators is 2 on average, even with fluctuations $\pm 2^{1 / 2}$ taken into account such a resonator will be present in a typical sample with a high probability. From Eq. (9) we readily find

$$
\tilde{Q}_{S}=\exp \left\{\left[\frac{\ln \left(S / 2 S_{0}\right)}{G}\right]^{1 / \lambda}\right\} .
$$

Finally, it is reasonable to assume that maximal $Q$-factors within the ensemble of samples lie within the interval between $\tilde{Q}_{S}$ and $Q_{S}$, which yields the following estimate for the distribution width in the logarithmic scale

$$
\ln Q_{S}-\ln \tilde{Q}_{S} \approx \frac{\ln 2}{\beta_{s}}
$$

where

$$
\beta_{s}=\lambda G^{1 / \lambda}\left[\ln \left(S / S_{0}\right)\right]^{1-\lambda^{-1}} .
$$

\section{Derivation of Eq. (1)}

The disorder configurations constituting high- $Q$ resonators are sparse, so that different resonators are statistically independent. Then the distribution function of the number, $N(Q, S)$, of resonators with quality factor in the interval $[Q, Q+d Q]$ within the area $S$ is Poissonian

$$
P_{n_{Q}}(N)=\frac{n_{Q}^{N} e^{-n_{Q}}}{N !}
$$

where $n_{Q}=S \rho(Q) d Q$ is the average number of resonators. Then probability, $F_{S}(Q)$, to find the resonator with the maximum quality factor in the interval $[Q, Q+d Q]$ is determined by the expression

$$
F_{S}(Q) d Q=P_{n_{Q}}(1) \prod_{Q_{1}>Q} P_{n_{Q_{1}}}(0),
$$

so that there is one resonator with quality factor from $Q$ to $Q+d Q$ and there are no resonators with the higher quality factor. Substituting Eq. (13) into Eq. (14), we obtain 


$$
F_{S}(Q) d Q=n_{Q} e^{-n_{Q}} \prod_{Q_{1}>Q} e^{-n_{Q_{1}}}=n_{Q} \exp \left(-\sum_{Q_{1} \geq Q} n_{Q_{1}}\right) .
$$

The summation in Eq. (15) goes over all the intervals $\left[Q_{1}, Q_{1}+d Q\right]$ with $Q_{1}>Q$. Replacing the sum in Eq. (15) by the integral, we get the following expression for the distribution function ${ }^{18}$

$$
F_{S}(Q)=S \rho(Q) \exp \left[-S \int_{Q}^{\infty} d Q_{1} \rho\left(Q_{1}\right)\right]
$$

It is easy to see that $F_{S}(Q)$ is normalized. To evaluate the integral in Eq. (16), we take into account that the density of resonators is a rapidly decreasing function of $Q$. Then the main contribution to the integral in Eq. (16) comes from low limit, $Q_{1} \approx Q$. With $\rho(Q)$ given by Eq. (6), the integral (16) can be rewritten as

$$
\begin{aligned}
& S \int_{Q}^{\infty} d Q_{1} \rho\left(Q_{1}\right)=\frac{S}{S_{0}} \int_{Q}^{\infty} d Q_{1} \exp \left[-G\left(\ln Q_{1}\right)^{\lambda}\right] \\
& =\frac{Q S}{S_{0}} \int_{1}^{\infty} d q \exp \left[-G(\ln Q)^{\lambda}\left(1+\lambda \frac{\ln q}{\ln Q}\right)\right]=\left(\frac{Q S}{\lambda G S_{0}}\right) \frac{\exp \left[-G \ln ^{\lambda} Q\right]}{\ln ^{\lambda-1} Q} .
\end{aligned}
$$

Substituting Eq. (17) into Eq. (16), we obtain the following expression for $F_{S}(Q)$

$$
F_{S}(Q)=\frac{S}{S_{0}} \exp \left\{-G \ln ^{\lambda} Q-\left(\frac{Q S}{\lambda G S_{0}}\right) \frac{\exp \left[-G \ln ^{\lambda} Q\right]}{\ln ^{\lambda-1} Q}\right\} .
$$

As it follows from the qualitative considerations (Sect. II), the distribution function, $F_{S}(Q)$, has a sharp maximum at $Q \approx Q_{S} \approx \exp \left\{\left[\ln \left(S / S_{0}\right) / G\right]^{1 / \lambda}\right\}$. For this reason we present $\ln Q$ in Eq. (18) as $\ln Q=\ln Q_{S}+\ln \left(Q / Q_{S}\right)$, where $\ln \left(Q / Q_{S}\right) \ll \ln Q_{S}$. Then Eq. (18) takes the form

$$
F_{S}(Q)=\frac{S}{S_{0}}\left(\frac{Q}{Q_{S}}\right)^{-\beta_{s}} \exp \left\{-G \ln ^{\lambda} Q_{S}-\left(\frac{Q}{Q_{S}}\right)^{-\beta_{s}}\left(\frac{S Q_{S}}{\lambda G S_{0}}\right) \frac{\exp \left[-G \ln ^{\lambda} Q_{S}\right]}{\ln ^{\lambda-1} Q_{S}}\right\},
$$

where $\beta_{s} \gg 1$ is expressed through $Q_{S}$ as follows

$$
\beta_{s}=\lambda G \ln ^{\lambda-1} Q_{S} .
$$

It is seen from Eq. (19) that $F_{S}(Q)$ assumes a simple form

$$
F_{S}(Q)=\frac{\beta_{s}}{Q}\left(\frac{Q}{Q_{S}}\right)^{\beta_{s}} \exp \left[-\left(\frac{Q}{Q_{S}}\right)^{\beta_{s}}\right]
$$

if $Q_{S}$ satisfies the relation 


$$
\left(\frac{S Q_{S}}{\lambda G S_{0}}\right) \frac{\exp \left[-G \ln ^{\lambda} Q_{S}\right]}{\ln ^{\lambda-1} Q_{S}}=1 .
$$

On the other hand, this relation can be rewritten as

$$
G \ln ^{\lambda} Q_{S}-\ln \left(Q_{S} / \beta_{s}\right)=\ln \left(S / S_{0}\right) .
$$

Since we have $G \gg 1$, the second term in the l.h.s. can be considered as a small correction. Neglecting this correction, we immediately realize that $Q_{S}$, determined from the qualitative consideration, indeed satisfies the relation (22). Upon substituting the value of $Q_{S}$, given by Eq. (8), into Eq. (20) we arrive to the final expression (12) for parameter $\beta_{s}$.

As the threshold gain, $I$, is proportional to $Q^{-1}$, the distribution of thresholds over the samples follows from Eq. (21) upon transformation $F_{S}(Q) d Q=F_{S}(I) d I$ with $Q / Q_{S}=\left(I / I_{S}\right)^{-1}$. Then $F_{S}(I)$ takes the form Eq. (1).

Since the distribution $F_{S}(I)$ is broad, it is best characterized by the moments of $\ln I$ defined as

$$
M_{n}=\left\langle\ln ^{n} \frac{I}{\langle I\rangle}\right\rangle
$$

rather than by the moments of $I$. In Eq. (24) the value $\langle I\rangle$ is the average threshold, calculated from the distribution (1). It is given by Eq. (3). As it is shown in the Appendix, the moments $M_{n}$ can be calculated analytically. They are expressed through the derivatives of the digamma function $\Psi^{(k)}=d^{k} \Psi(x) /\left.d x^{k}\right|_{x=1}$. The first three moments are given by the following expressions

$$
\begin{gathered}
M_{1}=-\frac{\Psi(1)}{\beta_{s}}-\ln \left[\Gamma\left(1-\beta_{s}^{-1}\right)\right], \\
M_{2}=\frac{\Psi^{(1)}}{\beta_{s}^{2}}+M_{1}^{2}, \\
M_{3}=-\frac{\Psi^{(2)}}{\beta_{s}^{3}}+3 M_{1} M_{2}-2 M_{1}^{3} .
\end{gathered}
$$

Note that Eq. (25) yields Eqs. (4)-(5) upon substituting $\Psi(1)=-\gamma$.

\section{Discussion}

In our consideration we have assumed that the areal density of resonators with anomalously large $Q$-values of the eigenmodes (sometimes in the literature they are 
called quasimodes $^{19}$ or quasistates $\left.^{3}\right)$ has the form $\rho(Q) \propto \exp \left[-G(\ln Q)^{\lambda}\right]$. Below we argue that this form is generic. More precisely, the arguments leading to Eq. (6) are applicable to all the models of the two-dimensional disorder ${ }^{5,17,20,21}$ considered in the literature. These arguments are the following ${ }^{11,22}$

(i) Denote with $A$ the characteristic area occupied by the disorder configuration that traps the light during the time $\omega^{-1} Q$. The fact that the resonator mode is confined, while its frequency, $\omega$, is degenerate with the continuum inevitably results in the evanescent losses and corresponding limitation on the quality factor

$$
\ln Q=\frac{\omega A^{1 / 2}}{c f\{\delta \epsilon(\mathrm{r})\}},
$$

where we have roughly estimated the curvature of the perimeter of the fluctuation as $A^{-1 / 2}$; the functional $f\{\delta \epsilon(\mathrm{r})\}$ describes the actual fluctuation of the dielectric constant, $\delta \epsilon(\mathrm{r})$, within the resonator. It is important that regardless of the form of $f\{\delta \epsilon(\mathrm{r})\}$, it decreases with overall magnitude of the fluctuation, $\overline{\delta \epsilon}$.

(ii) The phase volume of the fluctuation trapping the light for more than $Q$ periods is zero. Small statistical deviations of the dielectric constant from $\delta \epsilon(\mathrm{r})$ would couple the trapped mode to the propagating modes and cause its very fast leakage. Therefore, in order to maintain the high quality factor, these statistical deviations must be suppressed. The probability of such a suppression can be estimated as follows. There are $\mathcal{M}=A \omega^{2} / 4 \pi^{2} c^{2}$ squares with a side equal to the light wavelength within the area of the resonator. Each square couples to the propagating modes independently. Thus the resonator mode would leak out of a typical square during the time $\tau_{l}=l / c$, where $l$ is the mean free path. Hence, the probability that for each square this time exceeds $\omega^{-1} Q$ is given by

$$
\mathcal{P}=\left(\frac{Q}{\omega \tau_{l}}\right)^{-\mathcal{M}}=\exp \left[-\frac{A \omega^{2}}{4 \pi^{2} c^{2}} \ln (Q / k l)\right] .
$$

Upon expressing $A$ from Eq. (28) and substituting into Eq. (29) we obtain

$$
\mathcal{P}=\exp \left[-f^{2}\{\delta \epsilon(\mathrm{r})\}(\ln Q)^{2} \ln (Q / k l) / 4 \pi^{2}\right] .
$$

(iii) the rest of consideration is model dependent. This is because in order to obtain the density of resonators that "survive" the statistical fluctuations, one has to average Eq. (30) over the model-dependent distributions $\delta \epsilon(\mathrm{r})$ of trapping configurations with some weight $\exp [-\mathcal{F}\{\delta \epsilon(\mathrm{r})\}]$. Important is, however, that $\mathcal{P}$ increases sharply with $\overline{\delta \epsilon}$ due to the factor $f$, whereas the probability of a trapping configuration, $\exp [-\mathcal{F}\{\delta \epsilon(\mathrm{r})\}]$, drops exponentially with increasing $\overline{\delta \epsilon}$. Hence, we conclude that the density of resonators 


$$
\rho(Q)=\int \mathcal{D}\{\delta \epsilon\} \exp [-\mathcal{F}\{\delta \epsilon(\mathrm{r})\}] \mathcal{P}\{\delta \epsilon(\mathrm{r})\}
$$

is determined by the saddle point of the functional integral Eq. (31). Finally, any reasonable forms of $f$ and $\mathcal{F}$ (say, power-law) yields Eq. (6), since in course of taking the saddle point, the power of $\ln Q$ in the exponent reduces compared to Eq. (30) and the large factor $G(k l)$ emerges in front of the logarithm.

\section{Conclusions}

\section{A. General Remarks}

(i) In the present paper we argue that the dependence of a typical threshold on the sample size is given by Eq. (2), and thus falls off with $S$ slower than the power law, in contrast to conclusion drawn in Ref. 13 on the basis of numerical simulations. As follows from Eq. (2), the dependence of $I_{S}$ on $S$ turns into a power law only in the limit $\lambda \rightarrow 1$. In the most advanced analytical calculation ${ }^{11}$ for the model of a smooth spatial fluctuations of the dielectric constant, the value of parameter $\lambda$ was found to be $\lambda=11 / 8$. Existing experimental results ${ }^{23}$ on the dependence of the threshold on the sample area were fitted to the power-law. If we neglect the difference between $\lambda^{-1}=8 / 11$ and 1 , then the power-law dependence emerging from Eq. (2) will assume the form $I_{S} \propto\left(S / S_{0}\right)^{-1 / G}$, where the disorder parameter $G \gg 1$ is defined through Eq. (6) and decreases with the concentration of scatterers, $n$. Analytical consideration of Ref. 11 yielded $G^{-1} \propto n^{3 / 4}$. The results of numerical simulations of Ref. 13 also suggest that the exponent $G^{-1}$ increases with $n$, however it is impossible to extract the actual dependence of $G^{-1}$ on $n$ from the numerics.

(ii) Consideration in the present paper was based on the assumption that all random resonators, corresponding to a given disorder configuration, have the same quality factor, $Q$, regardless of their position within the sample. This is by no means the case, if a periodic modulation of the dielectric constant is present in the sample along with the disorder. In particular, if the modulation is strong enough to create the photonic bandgap, then $Q$-values for resonators located at the center of the sample would be exponentially higher that for resonators close to the perimeter. For this reason it is not surprising that in simulations reported in Ref. 24 the dependence of the threshold on the sample size was found to be much faster than Eq. (2). However, aside from expression (2) for $I_{S}$, the general distribution (1) should apply to the disordered samples with underlying periodicity considered in Ref. 24. 


\section{B. Universal Relation Between the Cumulants}

On the qualitative level, experimental verification of the distribution (1) of the random lasing threshold, predicted in the present paper, would be an observation of the characteristic asymmetry of the measured hystogram (long tail towards high thresholds and abrupt cutoff towards low thresholds). On the quantitative level, after parameters $\left\langle I_{S}\right\rangle$ and $\beta_{s}$ are determined from the hystogram, there still remains a question how accurately a strongly asymmetric distribution Eq. (1) describes the experimental data. There exists a regular procedure to test quantitatively the agreement between the theoretical and experimental distributions, when both are broad. This procedure is based on the comparison of different cumulants of $\ln I$ determined from the experimentally measured hystogram. In particular, the second and the third cumulants must satisfy a certain universal relation, which we derive below.

We define the cumulants of the distribution $F_{S}(I)$ as follows

$$
C_{n}=\left\langle\left[\ln \frac{I}{\langle I\rangle}-\left\langle\ln \frac{I}{\langle I\rangle}\right\rangle\right]^{n}\right\rangle,
$$

so that they are directly related to the moments $M_{n}$ [Eq. (24)]. Then, upon rewriting $C_{n}$ in terms of $M_{n}$, we obtain for the first two cumulants

$$
\begin{gathered}
C_{2}=M_{2}-M_{1}^{2}=\frac{\Psi^{(1)}}{\beta_{s}^{2}}, \\
C_{3}=M_{3}-2 M_{1} M_{2}+2 M_{1}^{3}=-\frac{\Psi^{(2)}}{\beta_{s}^{3}},
\end{gathered}
$$

where the relations Eqs. (25)-(27) have been used. The higher order cumulants can be found following the similar procedure

$$
C_{n}=\left(-\frac{1}{\beta_{s}}\right)^{n} \Psi^{(n-1)} \text {. }
$$

Expressing disorder parameter $\beta_{s}$ through $C_{2}$ and substituting the result into the expression (35), we obtain the universal relation between the cumulants of the distribution (1)

$$
\frac{C_{n}}{C_{2}^{n / 2}}=(-1)^{n} \frac{\Psi^{(n-1)}}{\left[\Psi^{(1)}\right]^{n / 2}} .
$$

In particular, for $n=3 \mathrm{Eq}$. (36) yields the following relation between the third and the second cumulants 


$$
\frac{C_{3}}{C_{2}^{3 / 2}}=-\frac{\Psi^{(2)}}{\left[\Psi^{(1)}\right]^{3 / 2}}=\frac{2^{5 / 2} 3^{3 / 2} \zeta(3)}{\pi^{3}} \approx 1.139,
$$

which are easiest to extract from the experimental data.

In conclusion of this subsection we note that $C_{2}^{1 / 2} \approx 0.76 / \beta_{s}$, which characterizes the width of the distribution (1), is close to the estimate Eq. (11) obtained from qualitative consideration in Sect. II.

\section{Relation to the Statistics of the Lyapunov Exponents for the Fluctuation Tails in 1D}

There are two major physical arguments that determine the shape of the distribution Eq. (1). (i) The occurrence of a mode with anomalously high $Q$ is a rare event; (ii) Different random resonators are statistically independent. Using these arguments, and the very general form Eq. (6) of the average density $\rho(Q)$ leads directly to the distribution Eq. (1), as was demonstrated above.

Generality of the distribution Eq. (1) suggests that it might be applicable to the different situations when the net property of the finite-size sample is governed by the rare events. One such situation is the coherent transmission of a disordered 1D chain of a given length, $L$. For electron energy in the "body" of the band the distribution of log-transmission, reduces to the sum of random numbers, and, thus, is gaussian. Deep in the tail of the density of states this distribution is also close to gaussian, since the transmission, $T$, is dominated by "under-barrier tunneling" with a decrement weakly changing in space. Interference processes do not play a role in this energy interval. There exists, however, a range of energies close to the band-edge, where the central limit theorem is already not applicable, but the interference processes are still relevant. The distribution of the log-transmission becomes strongly asymmetric in this "fluctuation region" 26-28.

It might be conjectured that, similar to random lasing, the electron transmission within the fluctuation region is governed by the rare events. It is easy to identify these events, which are accidental formation of a "minibands" by different groups of almost even-spaced tail states ${ }^{10,25}$. One can also assume that different rare events are statistically independent. Unlike random lasing from the samples of a given area, this assumption is by no means trivial. However, if we adopt this assumption, then we immediately come to the conclusion that the transmission distribution is governed by Eq. (21), and thus can be described in a universal manner ${ }^{27}$. The analogy between the random lasing from a finite-area sample, and transmission of a long enough chain is based on the fact that lasing threshold is governed by the 
highest-quality resonator present in the sample, whereas the electron transmission occurs through the "most even-spaced" sequence of resonant tail states.

The validity of the conjecture about statistical independence of rare events within a long disordered chain can be checked by comparison of the consequences of this conjecture to the numerical results. Recently, the statistics of log-transmission coefficients (more precisely, of the Lyapunov exponents $\tilde{\gamma} \propto|\ln T|$ ) in the fluctuation tail of the Anderson model was studied numerically in Refs. 27, 28. On the basis of the simulations performed, the authors have found that the second, $\sigma^{2}=\left\langle[\tilde{\gamma}-\langle\tilde{\gamma}\rangle]^{2}\right\rangle$, and the third, $\varrho=\left\langle[\tilde{\gamma}-\langle\tilde{\gamma}\rangle]^{3}\right\rangle$, cumulants of the distribution can be approximately described by the following expressions

$$
\begin{gathered}
\tau=\sigma^{2} L l_{l o c}=D \kappa^{\alpha}+\tau_{m i n}, \\
\tau_{3}=\varrho L^{2} l_{l o c}=D_{3} \kappa^{\alpha_{3}}+\tau_{3, \min },
\end{gathered}
$$

where $l_{l o c}$ is the average localization length for an infinite chain, $\kappa=l_{\text {loc }} \sin [\pi \mathcal{N}(E)]$ is the scaling parameter, and $D, D_{3}, \alpha$, and $\alpha_{3}$ are numerical constants; $\mathcal{N}(E)$ is the number of states per unit length between the energy $E<0$ and the boundary $E=-2$ of the spectrum in the absence of disorder. Upon excluding $\kappa$ from Eqs. (38) and (39), we obtain

$$
\frac{\tau_{3}-\tau_{3, \min }}{\left(\tau-\tau_{\min }\right)^{\alpha_{3} / \alpha}}=\frac{D_{3}}{D^{\alpha_{3} / \alpha}}
$$

Assume now that the full distribution of the Lyapunov exponents is given by Eq. (21). This assumption suggests that the relation Eq. (40) is nothing but a different form of the relation Eq. (37). To check whether this is the case, we express $\tau$ and $\tau_{3}$ in terms of parameter $\beta_{s}$ as follows

$$
\begin{gathered}
\tau=\frac{\Psi^{(1)}}{\gamma \beta_{s}}, \\
\tau_{3}=\frac{\Psi^{(2)}}{\gamma \beta_{s}^{2}}<0 .
\end{gathered}
$$

Then the relation Eq. (37) takes the form

$$
\left|\frac{\tau_{3}}{\tau^{2}}\right|=-\frac{\gamma \Psi^{(2)}}{\left[\Psi^{(1)}\right]^{2}}=\frac{72 \gamma \zeta(3)}{\pi^{4}} \approx 0.51 .
$$

On the other hand, the simulations of Ref. 28 yielded the following values of the parameters $\alpha=0.27, \alpha_{3}=0.52, D_{3}=0.73, D=1.27$. Then for the ratio $\alpha_{3} / \alpha$ we 
get $\alpha_{3} / \alpha=1.93$, whereas the combination $D_{3} / D^{\alpha_{3} / \alpha}$ is approximately equal to 0.46 . We conclude that the numerical results of Ref. 28 satisfy the analytical relation (37) with high accuracy, suggesting that the distribution Eq. (21) might indeed describe the statistics of the coherent transmission coefficients through the tail of 1D Anderson chain. More conclusive judgment could be made if the moments higher than third were extracted from numerics of Ref. 28. In particular, the following analytical prediction for the fourth cumulant follows from the distribution (21) and Eqs. (A1), (A2) obtained using this distribution

$$
\frac{\tau_{4}}{\tau^{3}}=\frac{\gamma^{2} \Psi^{(3)}}{\left[\Psi^{(1)}\right]^{3}}=\frac{72 \gamma^{2}}{5 \pi^{2}} \approx 0.84
$$

Note in conclusion, that according to Eq. (21) the cumulants, $\tau_{i}$ should exhibit a logarithmical dependence of the length chain, $L$, through the parameter $\beta_{s} \propto$ $[\ln L]^{(\lambda-1) / \lambda}$. No such dependence was revealed in the simulations ${ }^{27,28}$. On the other hand two orders of magnitude change of $L$ in the simulations ${ }^{28}$ might be insufficient to uncover the above weak dependence, especially if $\lambda$ is close to 1 .

\section{Acknowledgments}

We acknowledge the support of the National Science Foundation under Grant No. DMR-0202790 and of the Petroleum Research Fund under Grant No. 37890-AC6.

\section{Appendix A: Moments of $F_{S}(I)$}

It is convenient to express the moments $M_{n}$ [Eq. (24)] of the distribution function $F_{S}(I)$ through the average of $\ln ^{k}\left(I / I_{S}\right)$ as follows

$$
M_{n}=\left\langle\ln ^{n} \frac{I}{\langle I\rangle}\right\rangle=\left\langle\left[\ln \frac{I}{I_{S}}+\ln \frac{I_{S}}{\langle I\rangle}\right]^{n}\right\rangle=\sum_{k=0}^{n} C_{k}^{n}\left\langle\ln ^{k} \frac{I}{I_{S}}\right\rangle\left(\ln \frac{I_{S}}{\langle I\rangle}\right)^{n-k},
$$

where $C_{k}^{n}$ are binomial coefficients. Taking into account the explicit expression Eq. (1) for $F_{S}(I)$, we can obtain the average of $\ln ^{k}\left(I / I_{S}\right)$ in terms of derivatives of Gamma function

$$
\begin{aligned}
\left\langle\ln ^{n} \frac{I}{I_{S}}\right\rangle & =\int_{0}^{\infty} d I F_{S}(I) \ln ^{n} \frac{I}{I_{S}}=\left(-\frac{1}{\beta_{s}}\right)^{n} \int_{0}^{\infty} d t e^{-t} \ln ^{n} t \\
& =\left.\left(-\frac{1}{\beta_{s}}\right)^{n}\left\{\frac{d^{n}}{d \epsilon^{n}} \int_{0}^{\infty} d t t^{\epsilon} e^{-t}\right\}\right|_{\epsilon \rightarrow 0}=\left.\left(-\frac{1}{\beta_{s}}\right)^{n}\left\{\frac{d^{n}}{d \epsilon^{n}} \Gamma(1+\epsilon)\right\}\right|_{\epsilon \rightarrow 0},
\end{aligned}
$$


where the variable $t=\left(I / I_{S}\right)^{-\beta_{s}}$ has been introduced in the intermediate integrals. It is convenient to rewrite the equation (46) through the digamma function $\Psi(x)=$ $d \ln \Gamma(x) / d x$ as follows

$$
\begin{aligned}
\left\langle\ln ^{n} \frac{I}{I_{S}}\right\rangle= & \left.\left(-\frac{1}{\beta_{s}}\right)^{n}\left\{\frac{d^{n}}{d \epsilon^{n}} \exp \left[\sum_{k=0}^{\infty} \frac{\Psi^{(k)}}{k !} \int_{0}^{\epsilon} d v v^{k}\right]\right\}\right|_{\epsilon \rightarrow 0} \\
& =\left.\left(-\frac{1}{\beta_{s}}\right)^{n}\left\{\frac{d^{n}}{d \epsilon^{n}} \exp \left[\sum_{k=0}^{\infty} \frac{\Psi^{(k)}}{(k+1) !} \epsilon^{k+1}\right]\right\}\right|_{\epsilon \rightarrow 0} .
\end{aligned}
$$

Here the abbreviations $\Psi^{(k)}=d^{k} \Psi(x) /\left.d x^{k}\right|_{x=1}$ have been introduced. Substituting Eq. (47) into Eq. (45), we obtain the expressions for the first three moments

$$
\begin{gathered}
M_{1}=-\frac{\Psi^{(0)}}{\beta_{s}}-\ln \left[\Gamma\left(1-\beta_{s}^{-1}\right)\right], \\
M_{2}=\frac{\Psi^{(1)}}{\beta_{s}^{2}}+M_{1}^{2},
\end{gathered}
$$

and

$$
M_{3}=-\frac{\Psi^{(2)}}{\beta_{s}^{3}}+3 M_{1} M_{2}-2 M_{1}^{3},
$$

where $\Psi^{(0)}=\Psi(1)=-\gamma, \Psi^{(1)}=\pi^{2} / 6$, and $\Psi^{(2)}=-2 \zeta(3)$. Here $\zeta(x)$ is the zeta-function.

${ }^{1}$ H. Cao, Y. G. Zhao, S. T. Ho, E. W. Seelig, Q. H. Wang, and R. P. H. Chang, Phys. Rev. Lett. 82, 2278 (1999).

${ }^{2}$ S. V. Frolov, Z. V. Vardeny, K. Yoshino, A. Zakhidov, and R. H. Baughman, Phys. Rev. B 59, R5284 (1999).

${ }^{3}$ H. Cao, Y. Ling, J. Y. Xu, C. Q. Cao, and P. Kumar, Phys. Rev. Lett. 86, 4524 (2001).

${ }^{4}$ R. C. Polson, A. Chipouline, and V. Vardeny, Adv. Mater. 13, 760 (2001).

${ }^{5}$ V. M. Apalkov, M. E. Raikh, and B. Shapiro, Phys. Rev. Lett. 89, 016802 (2002).

${ }^{6}$ A. L. Burin, H. Cao, G. C. Schatz, and M. A. Ratner, JOSA B, 21, 121 (2004).

${ }^{7}$ Note, that the fact that the "most capable" resonator is responsible for lasing in each sample, gives rise to the observed "rigidity" in the emission spectra of different samples, see R. C. Polson, M. E. Raikh, and Z. V. Vardeny, C. R. Acad. Sci. Paris IV, 3509 (2002).

${ }^{8}$ M. E. Raikh and I. M. Ruzin, Zh. Eksp. Teor. Fiz. 92, 2257 (1987) [Sov. Phys. JETP, 65, 1273 (1987)].

${ }^{9}$ M. E. Raikh and I. M. Ruzin, in Hopping and Related Phenomena, edited by H. Fritzsche and M. Pollak (World Scientific, Singapore 1990), pp. 217-242. 
${ }^{10}$ M. E. Raikh and I. M. Ruzin, in Mesoscopic Phenomena in Solids, edited by B. L. Altshuler, P. A. Lee, and R. A. Webb, (North Holland, Amsterdam 1991), pp. 303-353.

${ }^{11}$ V. M. Apalkov, M. E. Raikh, and B. Shapiro, in The Anderson Transition and its RamificationsLocalisation, Quantum Interference, and Interactions, "Lecture Notes in Physics" series, eds. T. Brandes and S. Kettemann, Springer Verlag, 630, 119 (2003).

${ }^{12}$ V. M. Apalkov, M. E. Raikh, and B. Shapiro, JOSA B 21, 132 (2004).

${ }^{13}$ A. L. Burin, M. A. Ratner, H. Cao, and R. P. H. Chang, Phys. Rev. Lett. 87, 215503 (2001).

${ }^{14}$ B. L. Altshuler, V. E. Kravtsov, I. V. Lerner, in Mesoscopic Phenomena in Solids, eds. B. L. Altshuler, P. A. Lee, and R. A. Webb (North Holland, Amsterdam, 1991).

${ }^{15}$ B. A. Muzykantskii and D. E. Khmelnitskii, Phys. Rev. B 51, 5480 (1995).

${ }^{16}$ V. I. Fal'ko and K. B. Efetov, Phys. Rev. B 52, 17413 (1995).

${ }^{17}$ A. D. Mirlin, Phys. Rep. 326, 259 (2000).

18 T. S. Misirpashaev and C. W. J. Beenakker, Phys. Rev. A 57, 2041 (1998).

${ }^{19}$ A. A. Chabanov, Z. Q. Zhang, and A. Z. Genack, Phys. Rev. Lett. 90, 203903 (2003).

${ }^{20}$ C. Vanneste and P. Sebbah, Phys. Rev. Lett. 87, 183903 (2001).

${ }^{21}$ P. Sebbah and C. Vanneste, Phys. Rev. B 66, 144202 (2002).

${ }^{22}$ V. M. Apalkov, M. E. Raikh, and B. Shapiro, Phys. Rev. Lett. 89, 126601 (2002).

${ }^{23}$ Y. Ling, H. Cao, A. L. Burin, M. A. Ratner, X. Liu, and R. P. H. Chang, Phys. Rev. A 64, 063808 (2001).

${ }^{24}$ A. Yamilov and H. Cao, Phys. Rev. A 69, 031803 (2004).

${ }^{25}$ I. M. Lifshits and V. Ya. Kirpichenkov, Zh. Eksp. Teor. Fiz. 77, 989 (1979) [Sov. Phys. JETP 50, 499 (1979)].

${ }^{26}$ H. Schomerus and M. Titov, Phys. Rev. E 66, 066207 (2002).

${ }^{27}$ L. I. Deych, M. V. Erementchouk, and A. A. Lisyansky, Phys. Rev. Lett. 90, 126601 (2003).

${ }^{28}$ L. I. Deych, M. V. Erementchouk, A. A. Lisyansky, A. Yamilov, and H. Cao, Phys. Rev. B 68, 174203 (2003).

${ }^{29}$ A. Yamilov and H. Cao, Phys. Rev. B 68, 085111 (2003).

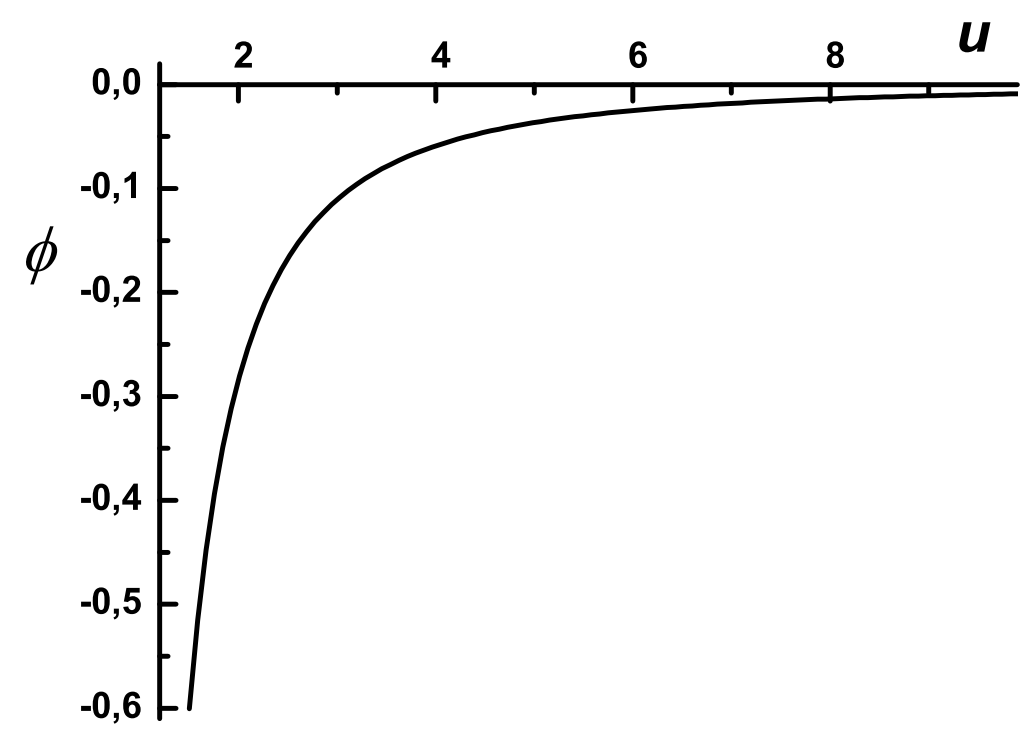

FIG. 1. Dimensionless function $\phi(u)$ is plotted from Eq. (5). 


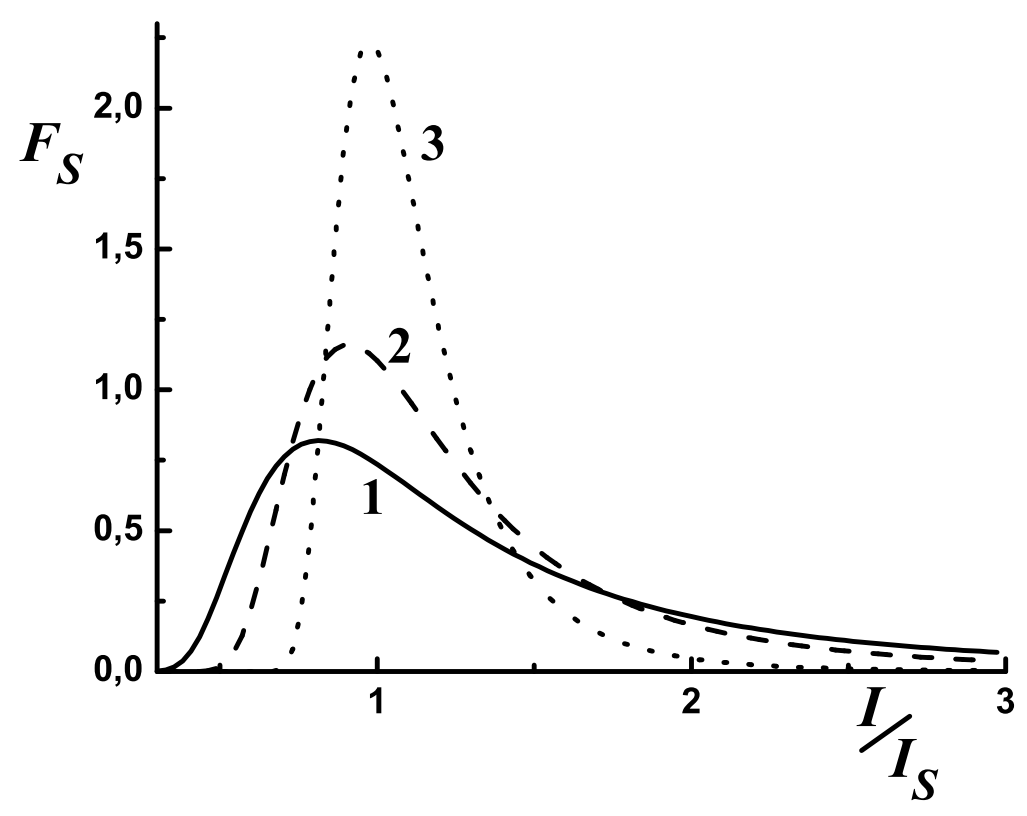

FIG. 2. Distribution function of the lasing thresholds over samples (excitation spots) calculated from Eq. (1) is plotted for different values of parameter $\beta_{s}$. Solid line: $\beta_{s}=2$; dashed line: $\beta_{s}=4$; dotted line: $\beta_{s}=6$. 